\title{
Reversible Bulk Oxidation of Ni Foil During Oscillatory Catalytic Oxidation of Propane: A Novel Type of Spatiotemporal Self-Organization
}

\author{
V. V. Kaichev, ${ }^{1,2 *}$ A. A. Saraev, ${ }^{1,2}$ A. Yu. Gladky, ${ }^{1}$ I. P. Prosvirin, ${ }^{1,2}$ R. Blume,${ }^{3,4}$ D. Teschner, ${ }^{3,4}$ \\ M. Hävecker, ${ }^{3,4}$ A. Knop-Gericke, ${ }^{4}$ R. Schlögl, ${ }^{3,4}$ and V. I. Bukhtiyarov ${ }^{1,2}$ \\ ${ }^{1}$ Boreskov Institute of Catalysis, Lavrentieva avenue 5, 630090 Novosibirsk, Russia \\ ${ }^{2}$ Novosibirsk State University, Pirogova street 2, 630090 Novosibirsk, Russia \\ ${ }^{3}$ Department of Heterogeneous Reactions, Max-Planck-Institute for Chemical Energy Conversion, \\ Stiftstraße 34-36, 45470 Mülheim an der Ruhr, Germany \\ ${ }^{4}$ Department of Inorganic Chemistry, Fritz Haber Institute, Faradayweg 4-6, D-14195 Berlin, Germany
}

(Received 28 March 2017; published 13 July 2017)

\begin{abstract}
A novel type of temporal and spatial self-organization in a heterogeneous catalytic reaction is described for the first time. Using in situ x-ray photoelectron spectroscopy, gas chromatography, and mass spectrometry, we show that, under certain conditions, self-sustained reaction-rate oscillations arise in the oxidation of propane over $\mathrm{Ni}$ foil because of reversible bulk oxidation of $\mathrm{Ni}$ to $\mathrm{NiO}$, which can be observed even with the naked eye as chemical waves propagating over the catalyst surface.
\end{abstract}

DOI: 10.1103/PhysRevLett.119.026001

Phenomena of temporal and spatial self-organization have been observed in many chemical and biological systems [1-3]. Extensive fundamental investigations of these phenomena began after the discovery of the wellknown Belousov-Zhabotinsky (BZ) reaction. Originally, the oscillations in this reaction were found in the 1950s by Belousov [4], who studied the oxidation of malonic acid by potassium bromate in a sulfuric acid solution using a Ce-based catalyst. He discovered that the mixture of reactants in this system oscillates in color between yellow and transparent. This unusual behavior was proved to be due to two competing chemical processes: the reduction of $\mathrm{Ce}$ (IV) ions by malonic acid to Ce(III) and the oxidation of $\mathrm{Ce}$ (III) back to $\mathrm{Ce}$ (IV) by bromate ions. The reaction can demonstrate up to several thousands of oscillatory cycles in a closed system. If ferroin is used in place of Ce-based catalysts, the color switches between blue and red. Nowadays, a wide range of similar homogeneous reactions, usually referred to as the $\mathrm{BZ}$ reaction, are known. Moreover, a number of recipes of "BZ cocktails" are available in the chemical literature and on the web that allow students to investigate the $\mathrm{BZ}$ reaction on their own. It is very important to note that in spatially nonhomogeneous systems, such as a simple Petri dish, the oscillations propagate as concentric colored chemical waves. Typical photos of this phenomenon have been published in the literature $[5,6]$.

In the 1970s, various phenomena of temporal and spatial self-organization were also found in the heterogeneous catalysis [7-9]. Generally, they can be related to selfsustained reaction-rate oscillations, which proceed in open systems far from equilibrium. For the first time, selfsustained kinetic oscillations were observed in the catalytic oxidation of $\mathrm{CO}$ over platinum [10] and in the catalytic oxidation of $\mathrm{H}_{2}$ over nickel [11]. To date, sinusoidal (harmonic) oscillations, relaxation-type oscillations, or oscillations with chaotic behavior have been discovered in approximately 40 catalytic reactions in a wide pressure range from ultrahigh vacuum up to atmospheric pressure and over many types of catalysts, including single-crystals, polycrystalline foils, wires, and supported catalysts [8]. The oscillations are observed even under steady flow conditions, and often they are accompanied by the propagation of spatiotemporal concentration patterns on the catalyst surface. For example, the spatiotemporal structures were observed in the oxidation of $\mathrm{CO}$ on $\mathrm{Pt}$ single-crystal surfaces under ultrahigh vacuum conditions. The oscillations are caused by the transitions of the surface between a highly reactive, predominantly oxygen-covered, state and a low reactive, CO-covered, state [7-9]. As a result of propagating reaction-diffusion fronts on the catalyst surface, there appear rotating spirals or irregular and rapidly changing structures denoted as "chemical turbulence" with a characteristic size of about several microns [11]. Because these chemical waves are due to the propagation of concentration distributions of adsorbed species on the catalyst surface, modern surface sensitive techniques such as photoemission electron microscopy are needed for their visualization $[12,13]$.

In contrast to the bulk chemical waves observed in the homogeneous $\mathrm{BZ}$ reaction, the chemical waves in the catalytic oxidation of $\mathrm{CO}$ are a surface phenomenon. As shown by Ertl [12], these surface chemical waves originate from migration of platinum atoms in the topmost layer of a single crystal, which accompany the adsorption and surface diffusion of $\mathrm{CO}$ and oxygen species. This model agrees well with a basic principle for all heterogeneous reactions; that is, at least one reactant undergoes a chemical 
transformation at an interface, in this case, on the surface of a solid catalyst. Herein, we describe a new type of spatiotemporal structure, which can form over solid catalysts at ambient pressure during self-sustained kinetic oscillations. These spatiotemporal structures are formed during self-sustained reaction-rate oscillations in the oxidation of propane over $\mathrm{Ni}$ foil, and can be observed with the naked eye. As the surface chemical waves cannot be observed without special techniques, these spatiotemporal structures are bulk related and must be due to changes in hundreds or even thousands of atomic layers of the catalyst. To elucidate the origin of this phenomenon, an in situ study was carried out using x-ray photoelectron spectroscopy (XPS), gas chromatography, and mass spectrometry (MS). All experiments were performed at the Innovative Station for In Situ Spectroscopy beam line at the synchrotron radiation facility BESSY II (Berlin, Germany). The station and the methods used are described in detail elsewhere [14-16].

The kinetic measurements were performed in a flow regime at a fixed total pressure of 0.5 mbar. A piece of 0.125-mm nickel foil (purity 99.99\%, Advent Research Materials Ltd.) was used as the catalyst. The regular relaxation-type oscillations arose under oxygen-deficient conditions, when the molar ratio of propane to oxygen ranged between $15: 1$ and $1: 1$. The period of oscillations varied from several to tens of minutes. The typical oscillatory patterns detected by mass spectrometry are presented in Fig. 1. One can see that most of the time the catalyst has low activity, and periodic peaks of $\mathrm{CO}, \mathrm{H}_{2}$, and $\mathrm{H}_{2} \mathrm{O}$ arise, indicating a transition of the catalyst to a high-active state. It should be noted that, under certain conditions, there appear some mixed-mode oscillations. For instance, when the molar ratio $\mathrm{C}_{3} \mathrm{H}_{8}: \mathrm{O}_{2}$ is $3: 1$, both large-amplitude oscillations and small-amplitude ones exist simultaneously. Most probably, this is a result of a perioddoubling bifurcation [17]. However, this effect requires additional mechanistic studies.

According to the on-line MS measurements, the conversion of propane oscillated in the range from $1 \%$ to $23 \%$. Gas chromatography detected also propylene together with $\mathrm{CO}, \mathrm{CO}_{2}, \mathrm{H}_{2}$, and $\mathrm{H}_{2} \mathrm{O}$ among the reaction products. It means that at least three catalytic reactions proceed when the $\mathrm{Ni}$ foil is heated in the reactant mixture: (i) the partial oxidation of propane to $\mathrm{CO}$ and $\mathrm{H}_{2}$, (ii) the total oxidation of propane to $\mathrm{CO}_{2}$ and $\mathrm{H}_{2} \mathrm{O}$, and (iii) the direct dehydrogenation of propane to propylene. In the moments when the conversion of propane is high, the selectivity toward $\mathrm{CO}$ achieves $96 \%-98 \%$, while the selectivity toward byproducts, such as $\mathrm{CO}_{2}$ and $\mathrm{C}_{3} \mathrm{H}_{6}$, is only $1 \%-3 \%$. In contrast, when the conversion is low, the selectivity toward $\mathrm{CO}$ is also low (in the range of 59\%-75\%) and the selectivity toward $\mathrm{CO}_{2}$ and $\mathrm{C}_{3} \mathrm{H}_{6}$ varies in the ranges of $8 \%-14 \%$ and $16 \%-30 \%$, respectively. Therefore, all three reactions proceed over the catalyst in the low-active state.

The oscillations of products and reactants in the gas phase were accompanied by synchronous changes in color of the Ni foil. A series of photos made during the oscillations is presented in Fig. 2. In the first photo, which corresponds to the low-active state, the color of the entire $\mathrm{Ni}$ surface is dark gray. In the second photo, a lighter spot appears at the center of the foil. The boundary of this light spot moves rapidly to the perimeter and, as a result, the entire surface of the Ni foil gets the bright metallic color, as

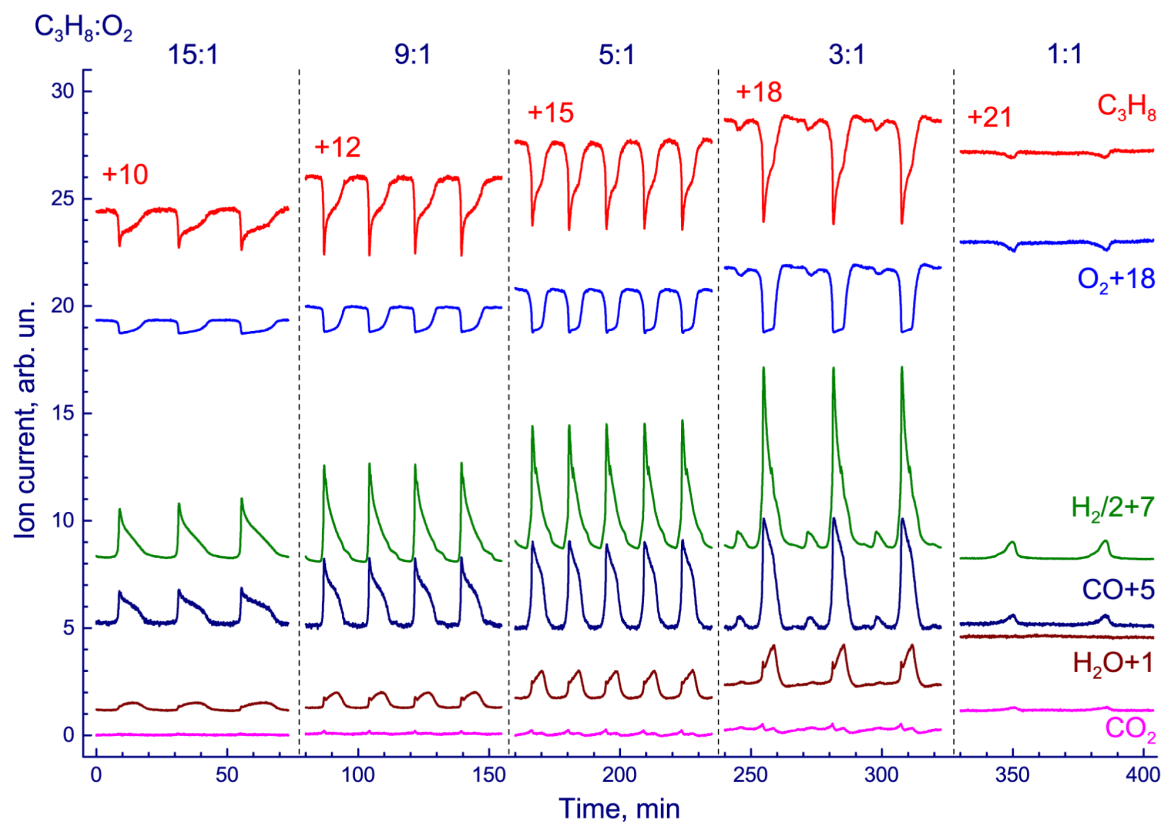

FIG. 1. Typical self-sustained oscillations in the oxidation of propane over $\mathrm{Ni}$ foil observed by mass spectrometry. The flow of $\mathrm{O}_{2}$ is fixed at $0.65 \mathrm{sccm}$, and the flow of $\mathrm{C}_{3} \mathrm{H}_{8}$ is varied. 


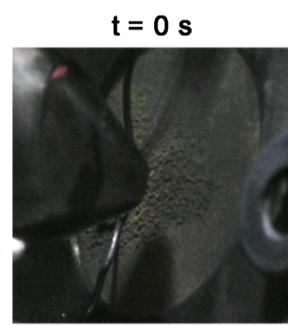

$\mathrm{t}=\mathbf{2 0 0 \mathrm { s }}$

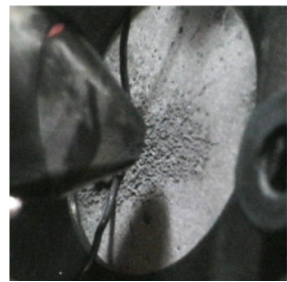

$t=100 s$

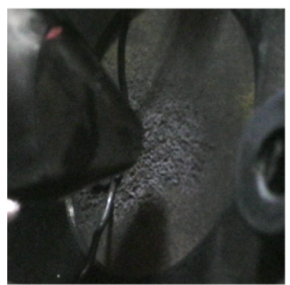

$t=230 s$

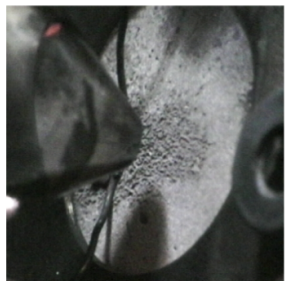

$t=110 s$

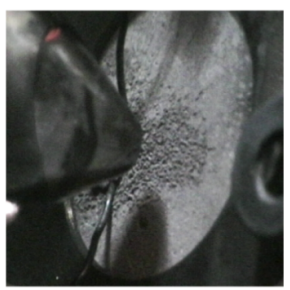

$\mathrm{t}=\mathbf{2 5 0 \mathrm { s }}$

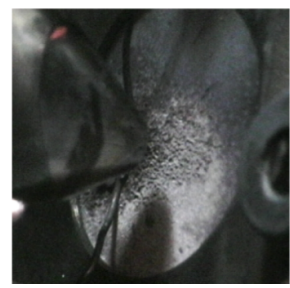

$t=150 s$

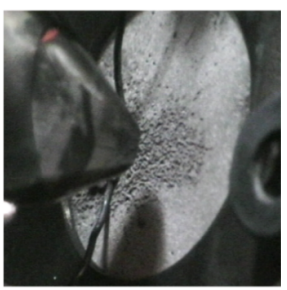

$t=800 s$

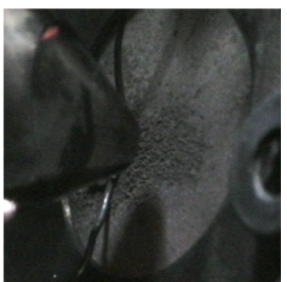

FIG. 2. Photos taken at some fixed intervals during the self-sustained oscillations. The molar ratio of propane to oxygen is 9: 1. The circle in the center of each photo, which changes its color, is a surface of Ni foil attached to a sample holder by a plate with a round hole with the diameter of approximately $8 \mathrm{~mm}$. One of fastening bolts (right side of the images), thermocouple wires, and a cone-shaped detail of an electronic lens system (left side) are also observed on the photos.

shown in the next two photos. The propagation rate of this wave is near $0.1 \mathrm{~mm} / \mathrm{s}$. This state is characterized by high conversion of propane. During the reverse process, the color is changing to dark gray and the boundary of the light spot moves with a lower rate from the perimeter of the foil to its center. The last photo of the Ni foil with dark gray color corresponds to the low conversion of propane. This process repeats itself regularly in time. A short movie is presented as the Supplemental Material [18].

This set of photos was synchronized with XPS measurements. Figure 3 shows the Ni2 $p_{3 / 2}$ and O1s core-level

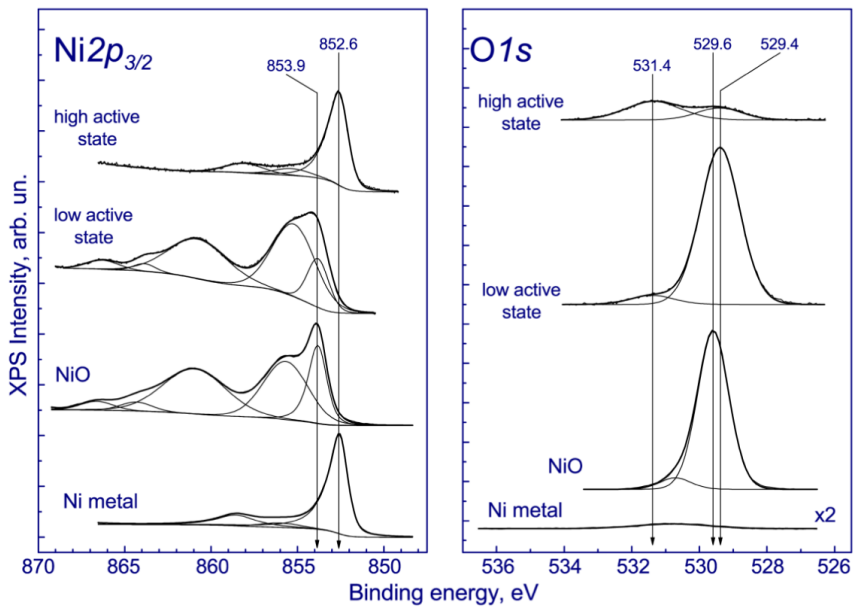

FIG. 3. Ni2 $2 p_{3 / 2}$ (a) and O1s (b) core-level spectra obtained in situ during the self-sustained oscillations in moments of low (near $2 \%$ ) conversion of propane ("low-active state") and high (near 18\%) conversion of propane ("high-active state"). The spectra of cleaned metallic $\mathrm{Ni}$ and of a $\mathrm{NiO}$ film grown by a treatment of the cleaned $\mathrm{Ni}$ foil in $\mathrm{O}_{2}$ at 1 bar for $10 \mathrm{~min}$ at $300^{\circ} \mathrm{C}$ are shown for comparison. spectra measured in situ from the Ni foil in low- and highactive states, which are compared with the corresponding spectra taken from the metallic $\mathrm{Ni}$ foil cleaned by $\mathrm{Ar}^{+}$ion bombardment for $30 \mathrm{~min}$ and from a thick $\mathrm{NiO}$ film. Analysis of the spectra indicates that the surface with the bright metallic color corresponds to nickel in the metallic state, while the change in color to dark gray is attributed to oxidation of nickel to $\mathrm{NiO}$. Indeed, the $\mathrm{Ni} 2 p_{3 / 2}$ spectrum of the Ni surface with the light color contains a sharp peak at $852.6 \mathrm{eV}$, which undoubtedly corresponds to metallic nickel. Two additional weak peaks at 855.5 and $859.0 \mathrm{eV}$ are also typical of metallic nickel and can be assigned to energy loss peaks due to plasmon excitations. A very similar spectrum is observed for the cleaned metallic $\mathrm{Ni}$ foil.

The change in color is accompanied by significant transformations in the $\mathrm{Ni} 2 p_{3 / 2}$ spectrum: there appear additional strong shakeup satellites and the main Ni2 $p_{3 / 2}$ peak shifts to a higher binding energy. Such a composite structure of the Ni2 $p_{3 / 2}$ spectrum is similar to that of bulk $\mathrm{NiO}$ (Fig. 3). It means that the drop in the rate of propane oxidation is due to the oxidation of nickel. This finding is confirmed by the $\mathrm{O} 1 s$ spectra. Indeed, the low-active state is characterized by a sharp peak at $529.4 \mathrm{eV}$, which can be assigned to oxygen species in the $\mathrm{NiO}$ lattice (the $\mathrm{O} 1 \mathrm{~s}$ spectrum of the $\mathrm{NiO}$ film contains a similar intense peak at $529.6 \mathrm{eV}$ ). The transition to the high-active state is accompanied by a significant decrease in the intensity of the $\mathrm{O} 1 \mathrm{~s}$ spectrum, indicating the reduction of nickel oxide. In this case, the O1s spectrum consists of two weak peaks at 529.4 and $531.4 \mathrm{eV}$, which can be attributed to chemosorbed oxygen species, and to $\mathrm{OH}$-groups or oxygen atoms dissolved in Ni [16].

These variations in the $\mathrm{Ni} 2 p_{3 / 2}$ and $\mathrm{O} 1 s$ spectra are reversible. Figure 4 shows the evolution of the intensities of 


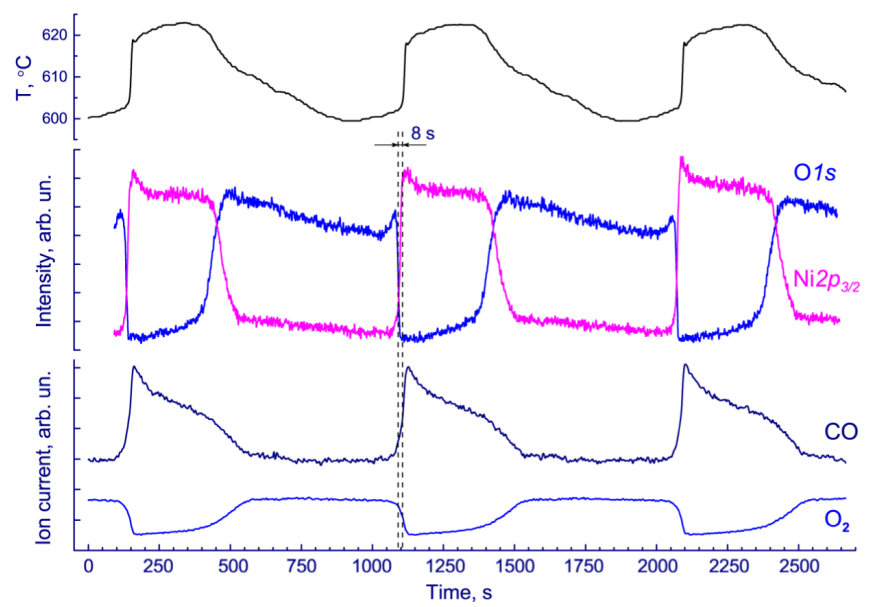

FIG. 4. Oscillations of the catalyst temperature and XPS signals at $529.5 \mathrm{eV}$ (the $\mathrm{O} 1 \mathrm{~s}$ binding energy of $\mathrm{NiO}$ ) and at $852.6 \mathrm{eV}$ (the $\mathrm{Ni} 2 p_{3 / 2}$ binding energy of metallic $\mathrm{Ni}$ ) observed simultaneously with the oscillations of gas-phase $\mathrm{O}_{2}(m / z=32)$ and $\mathrm{CO}(m / z=28) \mathrm{MS}$ signals during the oxidation of propane over Ni foil. The propane:oxygen ratio is $9: 1$.

the $\mathrm{Ni} 2 p_{3 / 2}$ peak at $852.6 \mathrm{eV}$ (metallic nickel) and the $\mathrm{O} 1 \mathrm{~s}$ peak at $529.5 \mathrm{eV}$ (lattice oxygen) measured in situ simultaneously with the gas-phase MS signals of consumed $\mathrm{O}_{2}$ and produced $\mathrm{CO}$. One can see that the XPS signals oscillate in antiphase. When the catalyst is in the highactive state (i.e., when the concentration of oxygen in the gas phase is low and the yield of $\mathrm{CO}$ is high), the $\mathrm{Ni} 2 p_{3 / 2}$ signal from metallic $\mathrm{Ni}$ reaches the maximum and the $\mathrm{O} 1 \mathrm{~s}$ signal from nickel oxide reaches the minimum.

It was also found that the oscillations of concentration of products and reactants are accompanied by the variation of the catalyst temperature (Fig. 4). Because the sample was heated with a near-infrared laser working at a fixed power, these temperature oscillations cannot result from heating artifacts and, thus, they reflect the chemical reactions occurring on the catalyst surface [14]. Indeed, the partial oxidation of propane, which is the main reaction proceeding over the catalyst in the high-active state, is an exothermic process $\left(\mathrm{C}_{3} \mathrm{H}_{8}+1.5 \mathrm{O}_{2} \rightarrow 3 \mathrm{CO}+4 \mathrm{H}_{2}\right.$; $\left.\Delta H_{1000}^{0}=206.4 \mathrm{~kJ} / \mathrm{mol}\right)$. That is, the temperature peaks observed synchronously with the oxygen uptakes are the result of propane oxidation. These oscillations cannot cause the changes in the color of the Ni foil (Fig. 2) because the peak-to-peak amplitude is only approximately $25^{\circ} \mathrm{C}$. However, the existence of local overheating can determine the start point of the chemical waves. For example, the wave of the reduction of $\mathrm{NiO}$ starts at the center of the $\mathrm{Ni}$ foil because this is the hottest spot. In contrast, the wave of the oxidation of metallic Ni starts from the perimeter and moves to the middle of the foil. Nevertheless, this is not a thermokinetic phenomenon: the regular self-sustained oscillations are observed at various temperatures from $550^{\circ} \mathrm{C}$ to $850^{\circ} \mathrm{C}$ [19] and thus they appear because of some other reason rather than because of reaching a critical temperature.

In order to estimate the thickness of the $\mathrm{NiO}$ layer, the Ni2 $p_{3 / 2}$ spectra were taken using photons with the energy of 1050 and $1350 \mathrm{eV}$ (spectra not shown here). Despite the difference in the kinetic energy of emitted photoelectrons (200 and $500 \mathrm{eV}$ ) and, thus, the difference in the XPS information depth, no signals of metallic nickel in the Ni2 $p_{3 / 2}$ spectrum were observed in the moments of low activity. Taking into account that the inelastic mean-free path of electrons $(\lambda)$ with the kinetic energy of $500 \mathrm{eV}$ in $\mathrm{NiO}$ is $10.6 \AA[15]$ and the XPS information depth is $3 \lambda$, the thickness of the NiO layer should exceed $32 \AA$. Moreover, according to our SEM study [16], the appearance of the oscillations is accompanied by the formation of a rough and porous structure over the Ni foil with the thickness of approximately 10-20 $\mu \mathrm{m}$. The features of such a structure can be seen in the middle of the foil (Fig. 2). Hence, the presented data indicate that the spatiotemporal structures observed simultaneously with the self-sustained reactionrate oscillations originate from the reduction and oxidation of many upper layers (up to several microns) of the Ni foil.

The reasons causing the transition between the highactive and low-active states, i.e., between metallic $\mathrm{Ni}$ and $\mathrm{NiO}$, are not clear yet. One of the possible reasons was discussed by Hendriksen et al. [20], who proposed that the formation and annealing of defects, such as surface atomic steps, is a driving force for reduction-oxidation during oscillations in the oxidation CO over Pt. Using in situ XRD, they observed the processes of smoothening of the metal surface and roughening of the oxide during inactive and active half-periods of the oscillations, respectively. We suppose that defects can also exert decisive influence on the oscillatory behavior in the oxidation of propane over Ni. Indeed, the line shape of the Ni2 $p_{3 / 2}$ spectrum of the lowactive state differs from the line shape of the spectrum of perfect $\mathrm{NiO}$ (Fig. 3). According to ab initio calculations [21], the difference may result from the presence of oxygen vacancies in the $\mathrm{NiO}$ structure.

To the best of our knowledge, this is a new type of spatiotemporal structure in the heterogeneous catalytic reactions. The main feature of this phenomenon is the significant thickness of the near-surface layer of the catalyst that changes its chemical composition. It differs very much from the spatiotemporal structures observed previously in the oxidation of CO over Pt $[12,13]$. Because we observe a propagation of a bulk concentration front, which is similar to the chemical waves in the BZ reaction, we define this phenomenon as a novel type of chemical waves. This is in agreement with Ross et al. [22], who defined one of the types of chemical waves as "a front of concentration variations travels through space."

Certainly, to develop a detailed mechanism for the regular chemical waves, further studies are necessary. As is well known, in the BZ reaction, the diffusional flow of 
chemical compounds that induce autocatalytic reaction is the key factor for the wave propagation. In our system, which is heterogeneous, this mechanism cannot occur. Because it is not a surface phenomenon, the surface diffusion can hardly initiate the wave propagation. The gas-phase diffusion also cannot be the reason for the wave propagation: at these pressures, gas-phase molecules move in the regime of the molecular flow, which is much faster than the wave propagation velocities observed in our experiments. Moreover, the transition from the high-active state to the low-active state occurs when the partial pressure of $\mathrm{O}_{2}$ is minimal; this means that the gas-phase diffusion cannot initiate the transition of Ni to NiO. Most probably, the bulk diffusion of $\mathrm{Ni}$ and $\mathrm{O}$ atoms is the driving force of the observed spatiotemporal structures.

We can speculate that similar bulk chemical waves can arise in other heterogeneous catalytic reactions, such as oxidation of other light hydrocarbons over transition metals, where similar oscillations have been observed. The velocity of such chemical waves depends on the temperature, morphology, and chemistry of the catalyst. The driving force for these self-sustained reaction-rate oscillations is the reversible bulk oxidation of the catalyst [23]. The transition between the high-active state and the low-active state (for example, metallic $\mathrm{Ni}$ and $\mathrm{NiO}$ ) occurs as propagating chemical waves. The process may start from the dissolution of oxygen atoms in the near-surface layer of $\mathrm{Ni}$, leading to the formation of a solid solution of oxygen in nickel, which can transform into NiO. Most likely, the first seeds of the $\mathrm{NiO}$ phase appear in places with the highest temperature and then increase in size. The latter process is promoted by the bulk oxygen diffusion. Given that under reaction conditions the nascent $\mathrm{NiO}$ layer is highly defective, spontaneous reduction of the $\mathrm{NiO}$ layer may start when the concentration of defects exceeds some critical level.

This work was conducted within the framework of Budget Project No. 0303-2016-0001 for the Boreskov Institute of Catalysis. We thank the Helmholtz Zentrum Berlin for allocation of synchrotron radiation beam time.

*vvk@catalysis.ru

[1] G. Nicolis and I. Prigogine, Self-Organization in NonEquilibrium Systems (Wiley, New York, 1977).

[2] A. S. Mikhailov, Foundations of Synergetics I (Springer, Berlin, 1990).
[3] Spatio-Temporal Organization in Nonequilibrium Systems, edited by S. C. Muller and T. Plesser (Project Verlag, Dortmund, 1992).

[4] B. P. Belousov, in Oscillations and Traveling Waves in Chemical Systems, edited by R. J. Field and M. Burger (Wiley, New York, 1985).

[5] A. N. Zaikin and A. M. Zhabotinsky, Nature (London) 225, 535 (1970).

[6] K. I. Agladze, V. I. Krinsky, and A. M. Pertsov, Nature (London) 308, 834 (1984).

[7] G. Ertl, Adv. Catal. 37, 213 (1990).

[8] M. M. Slinko and N. I. Jaeger, Stud. Surf. Sci. Catal. 86, 1 (1994).

[9] R. Imbihl and G. Ertl, Chem. Rev. 95, 697 (1995).

[10] P. Hugo and M. Jakubith, Chem. Ing. Tech. 44, 383 (1972).

[11] V. D. Belyaev, M. M. Slinko, V. I. Timoshenko, and M. G. Slinko, Kinet. Catal. 14, 810 (1973).

[12] G. Ertl, Science 254, 1750 (1991).

[13] S. Nettesheim, A. von Oertzen, H. H. Rotermund, and G. Ertl, J. Chem. Phys. 98, 9977 (1993).

[14] A. Knop-Gericke, E. Kleimenov, M. Hävecker, R. Blume, D. Teschner, S. Zafeiratos, R. Schlögl, V. I. Bukhtiyarov, V. V. Kaichev, I. P. Prosvirin, A. I. Nizovskii, H. Bluhm, A. Barinov, P. Dudin, and M. Kiskinova, Adv. Catal. 52, 213 (2009).

[15] V. V. Kaichev, A. Y. Gladky, I. P. Prosvirin, A. A. Saraev, M. Hävecker, A. Knop-Gericke, R. Schlögl, and V. I. Bukhtiyarov, Surf. Sci. 609, 113 (2013).

[16] V. V. Kaichev, D. Teschner, A. A. Saraev, S. S. Kosolobov, A. Yu. Gladky, I. P. Prosvirin, N. A. Rudina, A. B. Ayupov, R. Blume, M. Hävecker, A. Knop-Gericke, R. Schlögl, A. V. Latyshev, and V. I. Bukhtiyarov, J. Catal. 334, 23 (2016).

[17] A. B. Zorin and Y. Makhlin, Phys. Rev. B 83, 224506 (2011).

[18] See Supplemental Material at http://link.aps.org/ supplemental/10.1103/PhysRevLett.119.026001 for observing the propagation of chemical waves over the catalyst surface during the self-sustained oscillations in the oxidation of propane.

[19] A. Yu. Gladky, V. V. Kaichev, V. K.Ermolaev, V. I. Bukhtiyarov, and V. N. Parmon, Kinet. Catal. 46, 251 (2005).

[20] B. L. M. Hendriksen, M. D. Ackermann, R. van Rijn, D. Stoltz, I. Popa, O. Balmes, A. Resta, D. Wermeille, R. Felici, S. Ferrer, and J. W. M. Frenken, Nat. Chem. 2, 730 (2010).

[21] M. A. van Veenendaal and G. A. Sawatzky, Phys. Rev. Lett. 70, 2459 (1993).

[22] J. Ross, S. C. Müller, and C. Vidal, Science 240, 460 (1988).

[23] A. A. Saraev, Z. S. Vinokurov, V. V. Kaichev, A. N. Shmakov, and V. I. Bukhtiyarov, Catal. Sci. Technol. 7, 1646 (2017). 